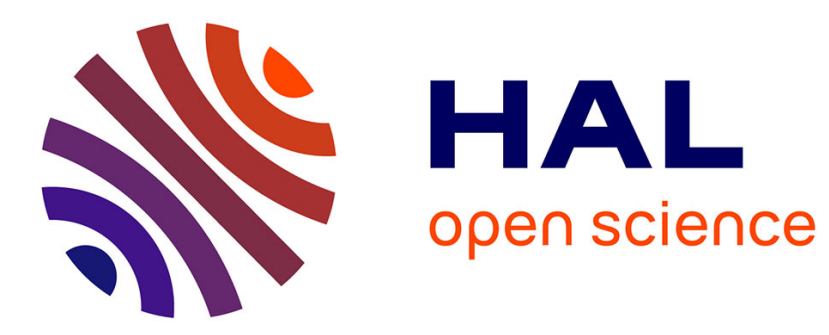

\title{
Générateur d'impulsions symétriques pour la mesure de la température d'électrons chauds
} Jean-Claude Portal, Salomon Askenazy, Gérard Bauzil

\section{To cite this version:}

Jean-Claude Portal, Salomon Askenazy, Gérard Bauzil. Générateur d'impulsions symétriques pour la mesure de la température d'électrons chauds. Revue de Physique Appliquée, 1969, 4 (4), pp.481-484. 10.1051/rphysap:0196900404048100 . jpa-00243315

\section{HAL Id: jpa-00243315 https://hal.science/jpa-00243315}

Submitted on 1 Jan 1969

HAL is a multi-disciplinary open access archive for the deposit and dissemination of scientific research documents, whether they are published or not. The documents may come from teaching and research institutions in France or abroad, or from public or private research centers.
L'archive ouverte pluridisciplinaire HAL, est destinée au dépôt et à la diffusion de documents scientifiques de niveau recherche, publiés ou non, émanant des établissements d'enseignement et de recherche français ou étrangers, des laboratoires publics ou privés. 


\title{
GÉNÉRATEUR D’IMPULSIONS SYMÉTRIQUES POUR LA MESURE DE LA TEMPÉRATURE D'ÉLEGTRONS GHAUDS
}

\author{
Par Jean-Claude PORTAl et Salomon ASKenazy, \\ Laboratoire de Physique des Solides, associé au G.N.R.S., Faculté des Sciences, ir8, route de Narbonne, 3 I-Toulouse, \\ et GÉrARd BAUZIL, \\ Laboratoire d'Automatique et de ses Applications Spatiales, du C.N.R.S., 31-Toulouse.
}

(Reçu le 23 juin 1969.)

\begin{abstract}
Résumé. - Nous décrivons un générateur d'impulsions symétriques qui utilise le principe de décharge d'une ligne à retard par l'intermédiaire d'un thyratron, adapté à l'étude des porteurs chauds dans les semiconducteurs.
\end{abstract}

Abstract. - We describe a symmetric pulse generator convenient for studying hot carriers properties in semiconductors. This generator uses a delay line discharged through a thyratron.

I. Introduction. - Dans le domaine concernant la technique de mesure de la température des électrons chauds, il existe une méthode basée sur l'effet thermoélectrique étendue au cas des porteurs chauds. La mesure de la tension due à l'effet thermoélectrique permet d'atteindre directement la température des électrons chauds en prenant un échantillon en forme de T [1], [2].

La réalisation de la chaîne de mesure [3] est facilitée, et la précision des mesures est accrue par l'utilisation d'un générateur d'impulsions symétriques dont les avantages sont :

- d'une part, de créer une masseflottante au voisinage des points de mesure 3 et 4 (fig. 1) et d'utiliser dans de bonnes conditions la chaîne de mesure,

- d'autre part, de permettre l'analyse répétée des mesures de tensions thermoélectriques, à l'aide d'un traducteur tension-temps qui élimine les erreurs aléatoires liées à une mesure unique.

II. Caractéristiques du générateur. — L'étude des électrons chauds dans les semiconducteurs nécessite, pour des échantillons d'impédance généralement faible, l'application d'impulsions de tension capables de créer un champ électrique dans la gamme de 30 à $800 \mathrm{~V} / \mathrm{cm}$. Lors de notre étude sur le silicium [3], les échantillons avaient une impédance égale ou supérieure à $125 \Omega$.

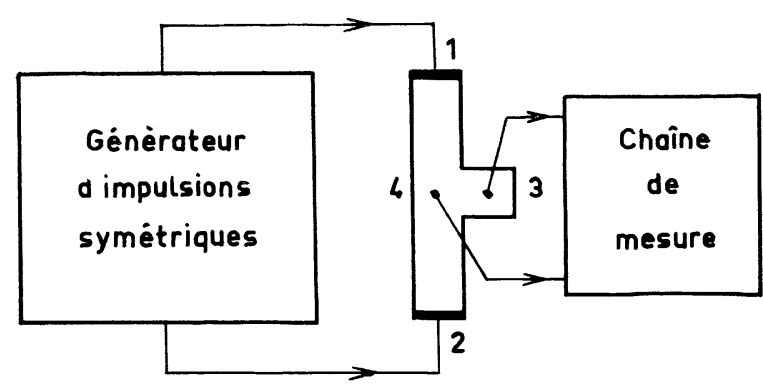

FIG. 1. - Schéma synoptique simplifié d'étude de la température des électrons chauds.

Dans ces conditions, la puissance crête $P$ dissipée dans un échantillon peut être au maximum de l'ordre de $5 \mathrm{~kW}$. Pour " chauffer » les électrons sans chauffer le réseau, nous devons limiter la puissance moyenne $\bar{P}_{\mathrm{m}}$ dissipable dans l'échantillon à $10^{-2} \mathrm{~W}$ (valeur correspondante à une élévation moyenne de température de $1 / 100^{\circ} \mathrm{C}$ pour les échantillons utilisés). Si $\tau$ est la durée de l'impulsion et $\nu_{r}$ la fréquence de récurrence, nous obtenons :

$$
\nu_{r} . \tau \leqslant \frac{\bar{P}_{\mathrm{m}}}{P}=2 \times 10^{-6} .
$$

Gette condition est satisfaite si $\nu_{r}=0,5 \mathrm{~Hz}$ avec $\tau \leqslant 4 \mu s$. 
III. Description du générateur. - 1. Principe. Ge générateur utilise comme principe une ligne à retard dont la décharge est commandée par un thyra-

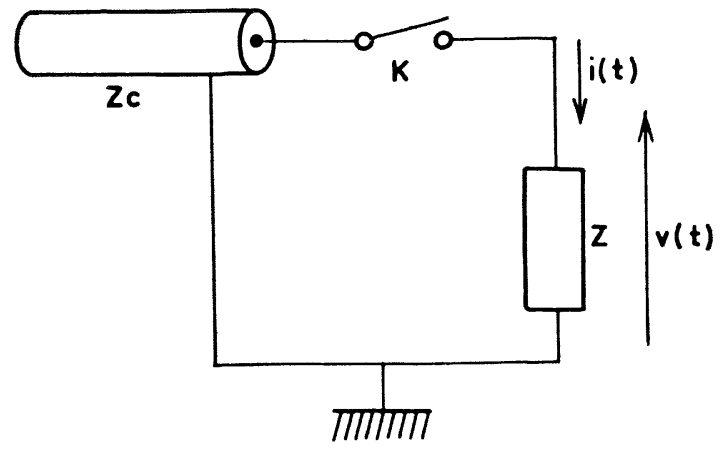

FIG. 2. - Principe du générateur d'impulsions.

tron à hydrogène jouant le rôle de l'interrupteur $\mathrm{K}$ (fig. 2).

La ligne à retard a une impédance caractéristique $Z_{\mathrm{c}}$ et une extrémité ouverte. Elle est chargée à la tension $V_{0}$. Elle se décharge dans une impédance extérieure $Z$ avec un courant $i(t)$ pouvant s'écrire [4] :

$$
\begin{aligned}
& i(t)= \frac{V_{0}}{Z_{\mathrm{c}}+Z}\{1-U(t-2 \delta) \\
&-\frac{Z_{\mathrm{c}}-Z}{Z_{\mathrm{c}}+Z}[U(t-2 \delta)-U(t-4 \delta)] \\
&\left.+\left(\frac{Z_{\mathrm{c}}-Z}{Z_{\mathrm{c}}+Z}\right)^{2}[U(t-4 \delta)-U(t-6 \delta)]-\ldots\right\}
\end{aligned}
$$

avec $U(\Delta t)=1$ pour $\Delta t>0$;

$U(\Delta t)=0$ pour $\Delta t<0$

$\Delta t=t-n \delta$ pour $n=2,4,6 \ldots$

Aux extrémités de $Z$ apparaît une tension :

$$
v(t)=Z i(t) \text {. }
$$

Le temps $\delta$ est le retard introduit par la ligne ou encore le temps de propagation d'un échelon de tension.

Dans le cas particulier où $Z=Z_{\mathrm{c}}$, la ligne se décharge à courant constant $I=\frac{V_{0}}{2 Z_{\mathrm{c}}}$ et à tension constante $V=\frac{V_{0}}{2}$ pendant le temps $\tau=2 \delta$.

Toute l'énergie initialement stockée dans la ligne à retard est dissipée dans la charge $Z$.

Pour obtenir deux impulsions de tension, symétriques par rapport à la masse, nous avons placé dans le circuit de décharge de la ligne à retard deux impédances de valeur égale à $\frac{Z_{\mathrm{c}}}{2}$ (fig. 3 ).

Les impulsions de tension obtenues ont une amplitude $\frac{V_{0}}{4}$ et une largeur $2 \delta$.

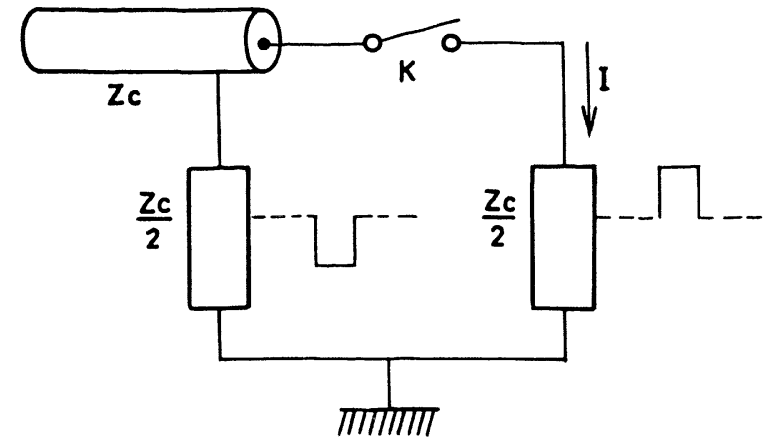

FIG. 3. - Principe du générateur d'impulsions symétriques.

2. RÉAlisation. - La ligne à retard est réalisée à l'aide d'un câble coaxial d'impédance caractéristique $Z_{\mathrm{c}}=125 \Omega$, de capacité $36 \mathrm{pF} / \mathrm{m}$ qui donne un affaiblissement de $0,1 \mathrm{~dB} / \mathrm{m}$ à $200 \mathrm{MHz}$. La longueur du câble est réglable par bonds de $100 \mathrm{~m}$ jusqu'à $500 \mathrm{~m}$ et permet d'obtenir des impulsions positive et négative de largeur ajustable par bonds de 800 ns jusqu'à $4 \mu s$.

L'adjonction d'une résistance variable aux extrémités de l'échantillon (d'impédance supérieure à $Z_{\mathrm{c}}$ ) permet d'adapter l'impédance du circuit de décharge de la ligne à retard et d'obtenir une masse flottante au voisinage des points de mesure 3 et 4 . A cause du non-alignement de ces contacts, la mesure de la tension due à l'effet thermoélectrique des électrons chauds nécessite une inversion de polarité du champ électrique appliqué à l'échantillon. Elle est réalisée par l'intermédiaire de relais commandés par un multivibrateur bistable. Le circuit de commande du thyratron permet d'obtenir une impulsion de déclenchement de $300 \mu \mathrm{s}$ de largeur avec une fréquence de répétition de $0,55 \mathrm{~Hz}$. Les relais sont commutés avant chaque amorçage du thyratron.

a) Fonctionnement du thyratron. - Le thyratron 2 D2 1 à hydrogène est utilisé en régime de commutation.

La ligne à retard se charge à travers les quatre résistances de $120 \mathrm{k} \Omega$ ( fig. 4) avec un courant de l'ordre du $\mathrm{mA}$ et avec une constante de temps de quelques ms. Elle se décharge par l'intermédiaire du thyratron avec un courant de l'ordre de l'ampère. En fin de décharge, la tension entre anode et cathode passe de la tension d'arc (de l'ordre d'une dizaine de volts) à une tension minimum d'arc (de l'ordre de $1 / 10$ de volt), maintenue pendant un temps de l'ordre de quelques dizaines de $\mu$ s et fonction des caractéristiques du thyratron [5]. L'extinction du thyratron se produit à la fin du palier de tension minimum d'arc.

Afin d'éviter un réamorçage avant que la ligne à retard soit complètement chargée, il faut, à la fin du palier de tension minimum d'arc, que la tension entre anode et cathode soit inférieure à la tension d'ionisation 


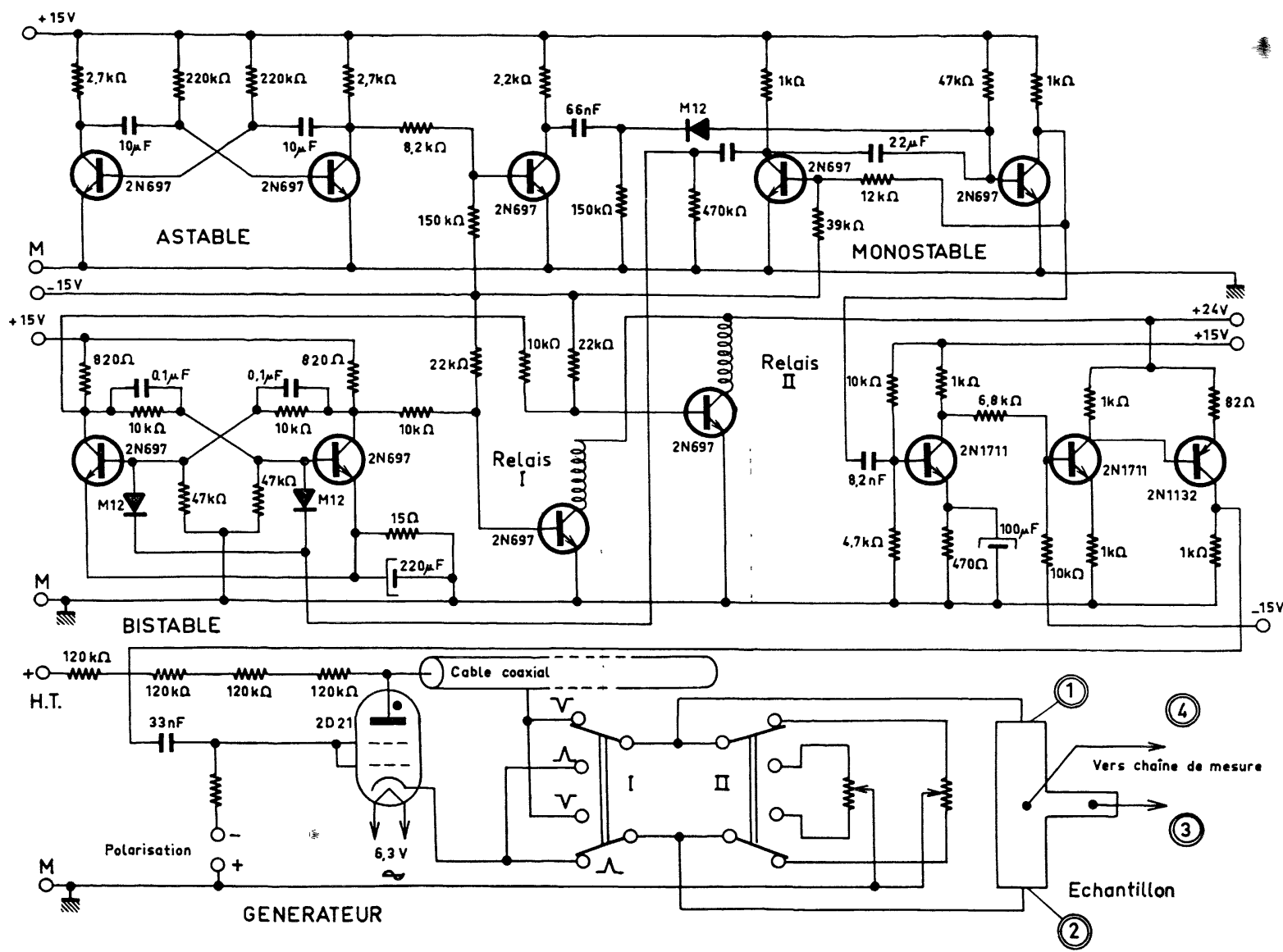

Fig. 4. - Schéma du générateur et des circuits de commande.

du gaz de remplissage. Cela est réalisé car le potentiel anodique augmente avec une constante de temps plus élevée que la largeur de l'impulsion de commande dont la fréquence de répétition est de $0,55 \mathrm{~Hz}$.

b) Forme des impulsions. - Les figures 5 et 6 donnent la forme des impulsions obtenues aux extrémités de l'échantillon, après adaptation de son impédance.

La longueur de câble coaxial est de $300 \mathrm{~m}$.

FIG. 5. - Forme des impulsions appliquées à l'échantillon : Amplitude $15 \mathrm{~V}(5 \mathrm{~V} / \mathrm{cm} ; 0,5 \mu \mathrm{s} / \mathrm{cm})$ :

Photographie 1 : Entre le contact 1 et la masse à l'instant $t_{0}$.

Photographie 3 : Entre le contact 1 et la masse à l'instant $t_{0}+1,8 \mathrm{~s}$.

Photographie 2 : Entre le contact 2 et la masse à l'instant $t_{0}$.

Photographie 4 : Entre le contact 2 et la masse à l'instant $t_{0}+1,8 \mathrm{~s}$.
Le temps de montée, limité au temps d'ionisation de l'hydrogène, est bref.

Le temps de descente légèrement plus important est
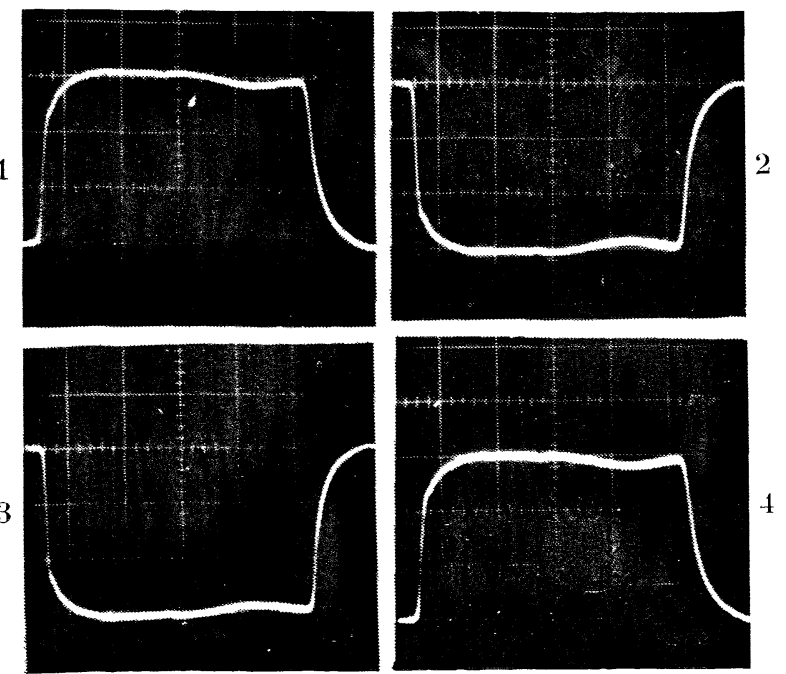

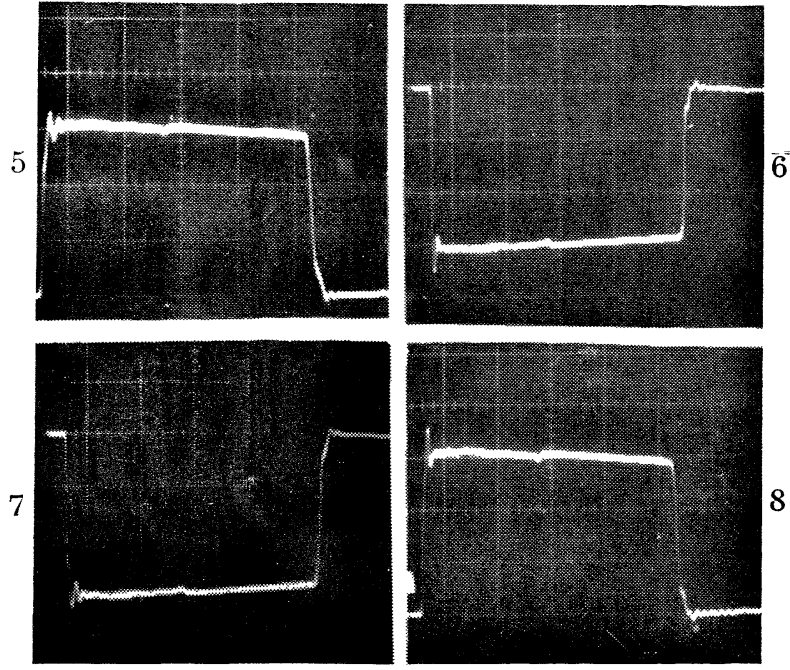

FIG. 6. - Forme des impulsions appliquées à l'échantillon : Amplitude $150 \mathrm{~V}(50 \mathrm{~V} / \mathrm{cm} ; 0,5 \mu \mathrm{s} / \mathrm{cm})$.

Photographie 5 : Entre le contact 1 et la masse à l'instant $t_{0}$.

Photographie 7 : Entre le contact 1 et la masse à l'instant $t_{0}+1,8 \mathrm{~s}$

Photographie 6 : Entre le contact 2 et la masse à l'instant $t_{0}$.

Photographie 8 : Entre le contact 2 et la masse à l'instant $t_{0}+1,8 \mathrm{~s}$. lié étroitement aux caractéristiques du câble et au temps écoulé pour que le potentiel anode diminue de la tension d'arc à la tension minimum d'arc.

Ce générateur utilisant un thyratron type 2 D2 1 a permis d'obtenir des impulsions symétriques atteignant une amplitude maximale de $2 \times 400$ volts.

IV. Conclusion. - Le générateur décrit nous a permis de mesurer dans de bonnes conditions la température des électrons chauds sur le silicium. Nous renvoyons, pour les résultats, aux articles cités en références [3] et [6].

Les échantillons examinés avaient une résistance supérieure à $125 \Omega$. En utilisant une ligne à retard d'impédance caractéristique faible, il est possible d'étudier des échantillons d'impédance inférieure à $125 \Omega$.

La fréquence de répétition des impulsions peut être modifiée dans une certaine gamme en faisant varier la période du multivibrateur astable.

L'effet d'affaiblissement de la ligne à retard sur l'amplitude de l'impulsion peut être réduit en utilisant un câble d'affaiblissement moindre en haute fréquence $(0,072 \mathrm{~dB} / \mathrm{m}$ à $400 \mathrm{MHz}$ Filotex $)$.

Il est possible d'obtenir des impulsions d'amplitude plus élevée en utilisant des thyratrons haute tension (krytrons-KN 22) supportant plusieurs kV en débitant un courant de quelques centaines d'ampères.

\section{BIBLIOGRAPHIE}

[1] STENBEK (M.), Izv. Akad. Nauk. S.S.S.R., 1956, 22 1560.

[2] BoK (J.), Thèse Doctorat ès Sciences Physiques, Université de Paris, 1959.

[3] Portal (J. C.), Thèse Doctorat de Spécialité (Physique du Solide), Université de Toulouse, 1969.
[4] White (H. J.), GILLETTE (P. R.) et LEBACQZ (J. V.), Pulse Generators, 175, McGraw-Hill, New York.

[5] Pham Huu Hiep, Thèse Doctorat ès Sciences Physiques, Université de Toulouse, 1963.

[6] Askenazy (S.), Portal (J. C.) et Crot (J.), C. $R$. Acad. Sci. Paris, 1969, 268, 1083. 